\title{
LA RECEPCIÓN DEL TEATRO DE FRANCISCO DE ROJAS ZORRILLA EN ITALIA. ALGUNAS ANOTACIONES*
}

\author{
ELENA E. MARCELLO \\ Universidad de Castilla-La Mancha
}

\begin{abstract}
Actualmente, las investigaciones acerca de la difusión del teatro aurisecular español en Italia viven un afortunado renacer, tanto en el ámbito del estudio de las piezas italianas hipertextualmente conexas con la producción española, como en el de la divulgación del teatro original. Estas averiguaciones no solo rescatan del olvido un ingente y variado corpus teatral, sino que ponen de manifiesto las dificultades metodológicas, bibliográficas y lingüísticas que las mismas encierran. Hace falta determinar las vías de transmisión del teatro español a Italia (el vaivén de compañías de un país a otro, los viajes de los dramaturgos españoles en Italia y viceversa, la edición colectiva o suelta de comedias, los contactos entre los dos países, etc.), indicar el tipo de relación que se establece con el modelo (traducción, imitación, refundición, sin olvidar las modificaciones implícitas en el traslado de un sistema dramático a otro o, incluso, de un género a otro, etc.), dar una justificación sociológico-cultural a la presencia/ ausencia de este teatro $\mathrm{y}$, finalmente, enfrentarse a los problemas lingüísticos de la adaptación a otro ambiente ${ }^{1}$.
\end{abstract}

\footnotetext{
* Este artículo se ha desarrollado dentro del Proyecto Edición de la obra dramática de Rojas Zorrilla I (HUM 2005-07408-C04-01-FILO), financiado por el Ministerio de Educación y Ciencia.
} 
Los indicios documentales sobre la recepción del teatro de Francisco de Rojas Zorrilla en tierras italianas son escasos, lacónicos y, a menudo, imprecisos. Solamente una profunda, detallada y exhaustiva indagación en las Bibliotecas y Archivos de las principales cortes italianas y del extranjero, similar a la que Marchante llevó a cabo para Calderón², podría asentar unas sólidas bases para la investigación sobre el tema. Por lo tanto, considérese este trabajo como un primer acercamiento a la cuestión.

\section{SIGLO XVII}

En el siglo XVII italiano, que promueve ese teatro «spagnoleggiante» despreciado por Croce y la crítica del siglo XX (por lo menos, hasta fechas recientes en las que se está paulatinamente re-conociendo y evaluando), el nombre del dramaturgo toledano aparece ligado a algunos miembros de la farándula de la Improvvisa y a comediógrafos de origen, principalmente, toscano y napolitano.

Según D'Antuono ${ }^{3}$, dentro de la producción anónima ligada a la commedia dell'Arte, La giustizia catalana, pieza que se conserva en el ms. 4186 de la Biblioteca Casanatense ${ }^{4}$ de Roma, podría remontarse a El Caín de Cataluña de Rojas. Como indica el título asignado para su catalogación, este códice constituye una Raccolta di scenari della commedia dell'Arte. En los ff. 93r-96r, se contiene un scenario titulado La giustizia catalana que tiene como protagonistas al «Conte di Barcellona/ Berlingerio primogenito/ Raimondo secondogenito/ Mag. ${ }^{\text {to }}$ Vice Conte/ finocchio/ frittellino servi/ Briselda Infanta di Tolosa/ Dama/ Rè d'Aragona/ Costante Infanta figlia/ Dottore consigliero/ Conte di Staffieri». Un examen del argumento confirma la adscripción propuesta. La pieza relata la historia de Berlingerio que quiere por mujer a la novia de su hermano Raimundo y termina cometiendo un fratricidio. En el final, recibe su castigo de mano del Conte, quien con los ojos vendados le sentenciará a muerte. A la hispanista citada anteriormente se le escapa que en el mismo manuscrito,

\footnotetext{
${ }^{1}$ Remito únicamente a Profeti, Maria Grazia, «La recepción del teatro áureo en Italia», en García Santo-Tomás, Enrique (ed.), El teatro del Siglo de Oro ante los espacios de la crítica. Encuentros y revisiones, Madrid-Frankfurt, Iberoamericana-Vervuert, 2002, pp. 427-457; a los trabajos sobre el tema promovidos por la misma hispanista y editados en Alinea Editrice, y a La comedia española y el teatro europeo del siglo XVII, eds. H. W. Sullivan, R. A. Galoppe, M. L. Stoutz, London, Tamesis, 1999.

2 Marchante, Carmen, "Calderón en Italia: traducciones, adaptaciones, falsas atribuciones y scenari», en VV. AA. Tradurre, riscrivere, mettere in scena, Firenze, Alinea Editrice, 1996, pp. 17-62.

${ }^{3}$ D'Antuono, Nancy, «La comedia española en la Italia del siglo XVII: la Commedia dell'Arte», en La comedia española y el teatro europeo del siglo XVII, 1999, pp. 1-36; 18.

4 Cairo, Laura - Quilici, Piccarda, Biblioteca teatrale dal '500 al '700. La raccolta della Biblioteca Casanatense, Roma, Bulzoni, 1981, 2 vols.
} 
La recepción del teatro de Francisco de Rojas Zorrilla en Italia. Algunas anotaciones.

en los ff. 155r-158v, existe otro scenario anónimo, titulado Il fratricida crudele, le finte caccie con Bertolino impiccato, que cuenta con los mismos protagonistas e historia. De modo que deben contabilizarse dos transposiciones al sistema dramático de la Improvvisa relacionadas con El Caín de Cataluña de Rojas Zorrilla.

El primer nombre asociado al de Rojas Zorrilla es el de Giacinto Andrea Cicognini (1606-finales de 1650/principios de 1651), hijo de aquel Iacopo también dramaturgo. Se trata de una de las figuras más celebradas del teatro italiano de la época, por su estro y habilidad en imitar el teatro español (con piezas derivadas de Calderón, Tirso, Lope de Vega, Villegas, etc.) innovando, a la vez, el autóctono. Las áreas geográficas de acción de Cicognini son Florencia, su ciudad natal, y Venecia, pero el éxito de sus obras promueve la difusión impresa en las principales ciudades de Italia y su representación. Entre su producción figura la comedia Il maritarsi per vendetta que es una versión de Casarse por vengarse de Rojas ${ }^{5}$. Varias son las ediciones de esta comedia: Il maritarsi per vendetta, Venetia, Giacomo Batti, 1662; Venetia, s. i. [Bartolomeo Lupardi], s. a. [finales 1662]6; Bologna, Carl'Antonio Peri, 1665; Venezia, Zaccheria Conzatti, 1668, etc. Ireneo Sanesi ${ }^{7}$ avanzaba la hipótesis de que «forse

\footnotetext{
${ }^{5}$ Cf. Sìmini, Diego, «Casarse por vengarse di Rojas Zorrilla nella traduzione di Giacinto Andrea Cicognini: Maritarsi per vendetta», en VV. AA., Tradurre, riscrivere, mettere in scena, Firenze, Alinea, 1996, pp. 95-116; Marcello, Elena E., «Appunti sulla fortuna del teatro spagnolo in Italia: Il maritarsi per vendetta di G. A. Cicognini», en El teatro italiano. Actas del VII Congreso de Italianistas, ed. Joaquín Espinosa, Valencia, Universitat de Valencia, 1998, pp. 399-406.

6 Al catalogar este ejemplar «romano», Franchi informa que «Il libraio romano Bartolomeo Lupardi, con bottega in piazza Navona all'insegna della Pace, procuratosi (probabilmente a Venezia) un consistente gruppo di manoscritti di opere di G. A. Cicognini, le pubblicò iniziando con questa tragicommedia. In seguito, raccolse in sette volumi tutti i testi cicogniniani da lui editi (non si tratta però di ristampe, ma di semplici unioni in volume delle opere già stampate con la sola aggiunta di un frontespizio generale). Lupardi, attivo a Roma almeno dal 1655 come autore di relazioni, pubblicò le sue edizioni fino al 1680 facendole stampare a Venezia, Bologna, Roma, Viterbo, Bracciano, Ronciglione, Terni e Todi da diversi stampatori o da proprie tipografie [...]. Questa edizione del Maritarsi per vendetta è databile alla fine del 1662 e riproduce quella veneziana di pochi mesi prima (In Venetia, M.DC.LXII. Appresso Giacomo Batti). Fonte dell'opera è la tragedia Casarse por vengarse di Francisco de Rojas y Zorrilla» (Franchi, Saverio, Drammaturgia romana. Repertorio bibliografico cronologico dei testi drammatici pubblicati a Roma e nel Lazio. Secolo XVII, Roma, Edizioni di Storia e Letteratura, 1988, p. 351). Sobre la imprenta dramática romana, véase también Franchi, Saverio, Le impressioni sceniche. Dizionario biobibliografico degli editori e stampatori romani e laziali di testi drammatici e libretti per musica dal 1579 al 1800, Roma, Edizioni di Storia e Letteratura, 1994; Franchi, Saverio, Drammaturgia romana II (1701-1750). Annali dei testi drammatici e libretti per musica pubblicati a Roma e nel Lazio dal 1701 al 1750, con introduzione sui teatri romani nel Settecento e commento storico-critico sull'attività teatrale e musicale romana dal 1701 al 1730, Ricerca storica, bibliografica ed archivistica condotta in collaborazione con Orietta Sartori, Roma, Edizioni di Storia e Letteratura, 1997.

${ }^{7}$ Sanesi, Ireneo, La commedia, Milano, Vallardi, 1944, 2 vols.; II, p. 178.
} 
dalla stessa commedia trae lo spunto iniziale, facendogli però seguire uno svolgimento del tutto diverso (sicché sarà da pensare piuttosto a un'altra fonte rimasta fin ora ignota), La forza del fato o Il matrimonio nella morte». En efecto, coincide en el núcleo inicial del enredo, que tiene como protagonistas al rey Alfonso de Castilla, Deianira, don Ferdinando y Rosaura. El rey, enamorado de Deianira, tiene que casarse con Rosaura para subir al trono; por su parte, la dama abandonada cede su mano a otro caballero. Sin embargo, el rey insiste en cortejar a la amada, que, para salvaguardar su honor, termina causando la muerte de la reina y de su esposo. Tras estos delitos, los amantes originarios se casan. A falta de un análisis más detenido, no queda que corroborar la intuición del ilustre italianista ${ }^{8}$, pero recordando que el matrimonio impuesto y el acceso al trono con ciertas condiciones, punto de partida de la pieza de Rojas, son recursos dramáticos extendidos en muchas «comedias trágicas» españolas.

Dentro de la producción atribuida al dramaturgo toscano figuran otros títulos que parecen anunciar un enredo muy al estilo español9. Piénsese, por ejemplo, en L'innocenza calunniata, overo la regina di Portogallo Elisabetta la santa (Bologna, Gioseffo Longhi, s. a. [probl. Entre 1655 y 1691]) ${ }^{10}$ que, por el tema dramatizado, podría relacionarse con Santa Isabel reina de Portugal de nuestro autor. En efecto, la comedia de Cicognini presenta ciertas coincidencias con la obra de Rojas, como, por ejemplo, los detalles del envío del ignorante mensajero a la muerte, de las flores de la reina, de la acusación de adulterio, etc. A la vez, son grandes las divergencias: presenta varios personajes diferentes, una acción más complicada tanto por el cruce de parejas como por la fuerte presencia de los criados, y centra la atención dramática en otros episodios de la historia de santa Isabel11. Queda, por lo tanto, la sospecha de que las dos piezas deban sus similitudes a una fuente común sobre el mito religioso más que a una relación directa. A falta de un estudio más detallado, la cuestión queda abierta.

Otra apertura hacia la comedia española manifiesta el más célebre Giulio Rospigliosi con La comica del cielo ovvero La Baldasara, representada el primer año de su pontificado como Clemente IX, es decir, en 1667. La obra se escribió durante la estancia de Rospigliosi en España como nuncio apostólico (1646-

\footnotetext{
${ }^{8}$ Como en su momento hizo Sìmini, «Casarse por vengarse...», p. 134 y ss.

${ }^{9}$ Intrigan, por ejemplo, las comedias I due prodigi ammirati overo Il privato favorito per forza e 'l prencipe infaticabile di sostenerlo, publicado en Bolonia, por Longhi, s. a. [prob. 1699] (Milán, Biblioteca Nazionale Braidense, Racc. Dram. 2409), La caduta del savio innamorato, editado en Macerata, por Grisei y Gioseppe Piccini, en 1667 (Milán, Biblioteca Nazionale Braidense, Racc. Dram. 0642) o Gl'equivoci dell'honore, overo la forza dell'honore, impreso en Bolonia, por los Herederos de Domenico Barbieri, en 1661 (Milán, Biblioteca Nazionale Braidense, Racc. Dram. 0230), etc.

${ }^{10}$ Se ha consultado el ejemplar de Milán, Biblioteca Nazionale Braidense, Racc. Dram. 1904.

${ }^{11}$ Se abre, por ejemplo, con el embrollo entre Lesbia, amante del rey, que acosa al hijo del mismo, Alfonso. De ahí, los celos y las sospechas del monarca, instigadas por el vasallo don Giovanni.
} 
La recepción del teatro de Francisco de Rojas Zorrilla en Italia. Algunas anotaciones.

1653) y la representación tuvo lugar durante el carnaval en el palacio romano de la familia Rospigliosi, con música de Anton Maria Abbatini y escenografía del Bernini $^{12}$. La fuente española, ya señalada y estudiada por Profeti13, es La Baltasara $^{14}$ de los tres ingenios Luis Vélez de Guevara, Antonio Coello y Francisco de Rojas Zorrilla.

Marco Napolioni parece ser quizás el mayor traductor y adaptador del teatro de Rojas Zorrilla. Lamentablemente, a las escasas informaciones sobre su vida y actividad se suma la desoladora situación de sus obras, casi todas sin localizar. Napolioni (o Napolione o Nobilione) es un cómico napolitano que se hizo famoso en las décadas centrales del siglo XVII por sus papeles de galán; de ahí, que se le conozca también como Flaminio o Flaminione, que era el nombre del personaje del «innamorato» que interpretaba. En 1640 este cómico tiene en alquiler con un tal Gregorio Chiaye el teatro de los Fiorentini de Nápoles, en el que solían recitar los españoles¹5; en 1647 está en Roma con una compañía que dirige en comunión con Carlo Cantù; poco después abandona la ciudad eterna para trasladarse de nuevo a Nápoles convenciendo a algunos actores de su compañía a seguirlo ${ }^{16}$. Entre ellos figura, probablemente, otra cómica destinada a traducir comedias españolas: Angiola d'Orso'17. El regreso en la ciudad natal coincide con la rebelión de Masaniello, con quien tenía lazos de amistad. Este último, conocedor de la fama de Napolioni, lo exhorta a entrar entre sus filas e, incluso, lo nombra embajador para la corte de España. Afortunadamente, el

12 Cf. Ademollo, Alessandro, I teatri di Roma nel secolo decimosettimo..., Roma, L. Pasqualucci, 1888 (reimpr.: Bologna, Forni, 1969), pp. 95-105; Kimiko Murata, Margaret, Opera for the Papal Court with texts by Giulio Rospigliosi, Tesis Doctoral, University of Chicago (UMI Dissertations Services), 1975, pp. 465-467.

13 Profeti, Maria Grazia, "Armi" ed "Amori": la fortuna italiana di Los empeños de un acaso», en VV. AA., Materiali, variazioni, invenzioni, Firenze, Alinea, 1996, pp. 99-120; 100; Profeti, Maria Grazia, «Dalla "Baltasara" alla "Comica del cielo": i meccanismi della scena nella scena», VV. AA., Percorsi europei, Firenze, Alinea, 1997, pp. 39-61.

14 Sobre esta comedia, además de los artículos citados de Profeti, cf. García de Enterría, María Cruz, «La Baltasara: pliegos, comedia y canción», en Symbolae pisanae. Studi in onore di Guido Mancini, ed. Blanca Periñán - Francesco Guazzelli, Pisa, Giardini, 1989, 2 vols., I, pp. 219-238; Stoll, Anita K., "La gran comedia de la Baltasara by "Tres ingenios de la corte"», Bulletin of the Comediantes, 48.2, 1996, pp. 329-338; García González, Almudena, «El espacio en la "Gran comedia de la Baltasara"», en Espacio, tiempo y género en la comedia española, Actas de las II Jornadas de teatro clásico (Toledo, 14, 15 y 16 de noviembre de 2003), ed. Felipe B. Pedraza, Rafael González Cañal y Gemma Gómez Rubio, Cuenca, Servicio de Publicaciones de la Universidad de Castilla-La Mancha, 2005, pp. 113-124.

15 Croce, Benedetto, I teatri di Napoli dal Rinascimento alla fine del secolo decimottavo, Bari, Laterza, 1916 (ahora: Milano, Adelphi, 1992), pp. 76-77.

${ }^{16}$ Cf. Prota-Giurleo, Ulisse, I teatri di Napoli nel 600. La commedia e le maschere, Napoli, Fausto Fiorentino, 1962 (reimpr.: Napoli, Il Quartiere, 2002), pp. 110-111, 136.

${ }^{17}$ Cf. Marigo, Marzia, "Angiola d'Orso, comica dell'Arte e traduttrice», en Biblioteca teatrale, n. 18, 1991, pp. 65-94; 66-67. 
actor, que no parece inclinado hacia la política, es destituido del encargo y puede volver a lo suyo, es decir, a las tablas. En agosto de 1657 está en la corte de Bolonia, pasa poco después en la de Florencia y, finalmente, en el teatro Samuele de Venecia. Luego, por razones todavía desconocidas, debe deshacer su compañía. Entonces, en julio de 1659 acepta entrar en la del duque de Módena desde el otoño hasta el carnaval ${ }^{18}$.

Las áreas de acción de Napolioni son Nápoles, Roma, Florencia (donde, según parece, el gran duque de Toscana Cosimo III le apreciaba mucho) y el ducado de Parma en un período de tiempo que abarca unos cuarenta años hasta 1680. Para estos escenarios el cómico napolitano traduce, adapta e interpreta un nutrido grupo de piezas dramáticas procedentes de España. Los testimonios documentales ${ }^{19}$, que equivocan con frecuencia la atribución del original español20, trasmiten las siguientes: Il gran catalan Sacralonga, Il Macometto, Il pericolo ne' rimedi, Il maritarsi per vendetta, Persile e Segismonda. Los títulos remiten a El catalán Serrallonga, El profeta falso Mahoma, Peligrar en los remedios, Casarse por vengarse, Persiles y Segismunda de Francisco de Rojas Zorrilla. Por lo que concierne a Il gran catalan Sacralonga $\mathrm{D}^{\prime}$ Antuono ${ }^{21}$ propone que la comedia italiana corresponda a la que fue compuesta por los tres ingenios Coello, Rojas y Vélez u otra, del mismo título, supuestamente escrita por Montalbán, Coello y Rojas, que no parece existir. En cambio, el $A B I$ enumera entre sus obras $I l$ maritarsi per vendetta, más conocida por la adaptación citada arriba de G. A. Cicognini, que también mencionan Peter $^{22}$ y MacCurdy ${ }^{23}$. Asimismo, atribuida a un «D. Ivan d'Allarion», se cita la comedia Gli aggravi trionfanti della gelosia, que, considerando los despistes de atribución de las comedias y la significación del título, quizás podría relacionarse con Donde hay agravios no hay celos del toledano.

18 Cf. Archivio Biografico Italiano (a partir de ahora, ABI), fiches da 619 a 778, da Mario Caio a Piccinini, vol. V, entrada Napolioni (Ejemplar consultado: Milán, Biblioteca Nazionale Braidense, Cons. B. 109); Indice Biografico Italiano (a partir de ahora IBI), ed. Tommaso Nappo, München, K. G. Saur, 2002, $3^{\circ}$ ed. revisada y corregida, 10 vols., VII, 2475; Leonelli, Nardo, Attori tragici e attori comici, Roma, Tosi, 1940, 2 vols., II, p. 411, 1-4.

19 Rasi, Luigi, I comici italiani. Biografia, Bibliografia, Iconografia, Firenze, Bocca, 1897-1905, 2 vols., II, pp. 174-175; Croce, I teatri di Napoli..., pp. 76-77, etc.

20 Atribuyen, por ejemplo, El perro del hortelano (Il cane dell'ortolano) a Mira de Amescua o Casas con dos puertas (La casa con due porte) a Juan Pérez de Montalbán.

${ }^{21}$ D'Antuono, «La comedia española en la Italia...», p. 29

22 Peter, A., Des Don Francisco de Rojas Tragödie "Casarse por vengarse" und ihre Bearbeitungen in den anderen literaturen, Dresda, Lehmannsche Buchdruckerei, 1898, cit. Sìmini, "Casarse por vengarse...», p. 99 , nota 5 .

23 MacCurdy, Raymond, Francisco de Rojas Zorrilla. Bibliografía crítica, Madrid, CSIC, 1965, p. 39. 
La recepción del teatro de Francisco de Rojas Zorrilla en Italia. Algunas anotaciones.

En la segunda mitad del siglo XVII debe colocarse la actividad teatral de la actriz Angela (o Angiola) d'Orso (pero también Dorsi, D’Orsi) ${ }^{24}$. Esta cómica, «prima Innamorata» de las compañías teatrales ligadas a los ducados de Parma (Farnese) y de Este (Bentivoglio), pisó los escenarios desde 1638, año en que se tiene noticia de una Angiolina actriz, hasta 1677. De toda su producción teatral, ampliamente dedicada a la labor de traducción-adaptación, se conservan actualmente cinco comedias: Di bene in meglio (1656), versión de Mejor está que estaba de Calderón; Con chi vengo vengo (1666), de Con quien vengo, vengo del mismo; Il finto medico (1669), que se publicó también con el título de Paolo Gemma overo Il ruffiano in Venezia e medico in Napoli, que deriva de El doctor Carlino de A. de Solís; Amore, onore e potere (1676), de Amor, honor y poder de Calderón.

El nombre de Angela d'Orso se une al de Francisco de Rojas Zorrilla por la comedia Il più improprio carnefice per la più giusta vendetta, probable traducción de El más impropio verdugo, que se anuncia como de próxima publicación en el año 1669, adelantándose así también el terminus ante quem trasmitido por D'Antuono ${ }^{25}$, es decir, 1672. Este título figura en el colofón de la edición de Il finto medico (Ronciglione, Toselli, 1669) al lado de otras piezas, todas sin localizar ${ }^{26}$. Se trata de un total de nueve comedias, para las que se avanzaron hipótesis sobre las fuentes por la sola semejanza de los títulos. Sin embargo, estas analogías pueden llevar a veces hacia terrenos pantanosos. Marigo $^{27}$ asocia A chi l'Onor li offende è gran pazzia se sente la puntur de gelosia de Angela d'Orso con No hay agravios como celos, si son los celos ofensa de Fernando de Frías y Santo. Sin embargo, este título podría afiliarse también a Donde hay agravios no hay celos de F. Rojas Zorrilla. De este atolladero podría librarnos solamente el hallazgo de las comedias y su comparación. Por poner otro ejemplo: la pieza Difendere l'inimico de la misma actriz, que tiene bastante cercanía nominal con otra de Carlo Celano (Proteggere l'inimico), parece anunciar el suceso representado en Obligados y ofendidos del dramaturgo toledano, es decir, el caso de un galán que se ve obligado a defender quien le agravió. En el caso de la cómica, Morigo 28 propone la comedia de Antonio de Solís Amparar al enemigo; mientras que en el de Celano, la lectura de la comedia desestima la hipótesis de Rojas. Queda por ver si se trata de otra versión de la de Solís y si tiene relación con la de Angela d'Orso.

\footnotetext{
${ }^{24} \mathrm{Cf}$. Dizionario Biografico degli italiani (a partir de ahora, DBI), Roma, Istituto della Enciclopedia Italiana, 1960-2005, 65 vols (hasta Lorenzetti), XLI, pp. 508-510; Marigo, «Angiola d'Orso...»; IBI, IV, p. 1330.

${ }^{25} \mathrm{D}^{\prime}$ Antuono, «La comedia española en la Italia...», p. 29.

${ }^{26}$ Marigo, «Angiola d'Orso...», pp. 70-71; Franchi, Drammaturgia romana..., 1988, p. 416.

27 Marigo, «Angiola d'Orso...», p. 70, nota 21.

${ }^{28}$ Marigo, «Angiola d'Orso...», p. 71.
} 
Más dibujada aparece la figura de Carlo Celano29, prolífico dramaturgo, nacido en Nápoles en 1610 (o, según otros, en 1617) y muerto en su ciudad natal en 1693. Su carrera forense se interrumpe al tomar partido por Masaniello, a causa del cual acaba en prisión. Después de su liberación, emprende la carrera eclesiástica y en 1664 es canónigo de la catedral de Nápoles. Carlo Celano se dedica a la actividad teatral con el pseudónimo de Ettore (o Ettorre) Calcolone, publicando en su ciudad natal numerosas piezas: Sopra l'ingannator cade l'inganno, L'infanta villana, Il vero consigliere del suo proprio male, que deriva de La fuerza lastimosa de Lope y propone la conocida historia del Conde de Alarcos; Proteggere l'inimico (Nápoles, 1664), Nelle cautele i danni, Gl'inganni fedeli, Negli sdegni gli amori overo La carboniera, La pietà trionfante overo L'empietà domata, La forza della fedeltà, Dall'amore l'ardire (Nápoles, 1664), L'ardito vergognoso (Nápoles, 1676) que remite, como se alude en el prólogo ${ }^{30}$, a El vergonzoso en palacio de Tirso, Chi trionfa morendo overo S. Casimiro (Nápoles, 1676), Come dispone il cielo overo La forza del sangue, La forza della fedeltà, etc.

Actualmente, se asocia a Rojas Zorrilla solamente por la comedia Non è padre essendo re, publicada por primera vez en Nápoles, Novello de Bonis, en 1663; luego, también en Roma, Moneta, 1669. Franchi señala que esta edición romana se reimprimió también en Bolonia, Gioseffo Longhi, 167031. En el prólogo al «lettore amico» de 166932 se informa que la obra «Fù composta anni sono fra lo spatio di diece giorni, en el tempo d'vn Carnevale, perche fusse passato d'alcuni Gentilhuomini amici dell'autore con qualche trattenimento; (rappresentata tre volte) riuscì grata al gusto di tutti, che non vi restorno più plausi per celebrare la delicatezza del sapore» (f. $\mathrm{A}^{3} \mathrm{r}$ ).

Sin embargo, otras piezas podrían enlazar con la producción del toledano. Los protagonistas de Gli effetti overo Gli eccessi della cortesia, comedia en tres actos escrita para el cardenal Caracciolo y representada durante el carnaval de 1676, coinciden en parte con los de Obligados y ofendidos: «Conte di Belfiore./ Horatio suo creato./ Cinthia Figlia di Sinibaldo./ Fenice sua creata,/ Sinibaldo

29 Cf. DBI, XXIII, pp. 339-340; Sanesi, La commedia, II, pp. 205-209; IBI, III, p. 898; Vaiopoulos, Katerina, «Per una bibliografia delle opere drammatiche di Carlo Celano», en Temi cervantini a Napoli. Carlo Celano e "La Zingaretta", Firenze, Alinea, 2003, pp. 69-120. Vaiopoulos también tiene en prensa un artículo sobre la obra Non è padre essendo re de Celano, que se presentó en el congreso Il viaggio della traduzione, Firenze,13-16 de junio 2006.

30 En la edición consultada (Bologna, Per Gio. Recaldini, 1679), conservada en Milán, Biblioteca Nazionale Braidense (Racc. Dram. 0710), la obra está dedicada al Ex. Sr. «Don Domenico Martio, Carrafa, Pacecco, duca di Madalioni, Marchese di Arienzo, Conte d'Avellaneda, e Cerrito, etc.». En el prólogo, el autor se presenta como «imitatore di quest'Opera [...] composta da vno de' più affettuosi seruitori, che habbia l'Eccellenza vostra ben conosciuto, benche qui comparisca mascherato» [ff. $\left.\mathrm{a}^{3} \mathrm{r}-\mathrm{v}\right]$.

${ }^{31}$ Franchi, Drammaturgia romana..., p. 421.

32 Se ha consultado el ejemplar de Roma, Biblioteca Nazionale, 35.8.A.10.1. 
Padre di Cinthia, e di/ Consaluo./ Alfonso Amico dei Sinibaldo./ Pantosca suo creato Napolita-/ no,/ Cassandra Sorella del Conte di/ Belfiore./ Teodoro Cugino del Conte./ Picone Brauo./ Capitano di Giustitia./ Scrivano di Corte»33. Con una rápida lectura de la comedia se reconoce el armazón de esta obra de Rojas Zorrilla: dos galanes (el Conte y Consalvo) que están recíprocamente ofendidos y obligados. Por supuesto, en esta adaptación son muchos los factores que se modifican -los cambios usuales del traslado al sistema teatral italiano (uso de la prosa, división en escenas, adecuación al ambiente, plurilingüismo, etc.), la ambientación, los nombres de algunos personajes, los criados napolitanos que sustituyen a los graciosos, ciertas secuencias, etc.- y otros tantos los que se mantienen o varían. Entre ellos, piénsese, por ejemplo, en la dama Fénix de Obligados y ofendidos que aquí se transforma en Cintia, mientras que su nombre, Fenice, se atribuye a una criada; en el detalle del lugar de la emboscada para matar al conde que se convierte de «puente de Alcántara» en «ponte della Valle» (p. 115) no solo en pos de su adecuación al ambiente italiano; en la reducción de las escenas con los pícaros y ladrones, etc. Dejando para otra ocasión el análisis detallado de ambas piezas, considérese esta comedia de Celano otro ejemplo de la difusión de Rojas en Italia.

El florentino Mattias Maria Bartolommei Smeducci (Florencia, 14 de agosto de 1640-24 de diciembre de 1695) está en contacto con el ambiente literario y teatral desde su tierna infancia gracias al padre, Girolamo, miembro de varias academias y dramaturgo polivalente ${ }^{34}$. Conocido por ser quien proporciona un primer listado de las piezas auténticas de G. A. Cicognini, Bartolommei compone varias obras teatrales que se publican en varias ciudades de Italia: Amore opera a caso (Florencia, 1668), La Sofferenza vince Fortuna (Florencia, 1669), Le gelose cautele (Bolonia, 1669), Il finto marchese (Roma, 1676), La prudenza vince amore (Venecia, 1682), Amore non vuole inganni (Bolonia, 1697). Como suele ocurrir, a veces la misma pieza se edita con títulos diferentes. Es el caso de la comedia Le gelose cautele que, según declaración del mismo dramaturgo, deriva de otra «D. Francesco de Roches Comico tra gli Spagnuoli più rinomati di non ultimo grido» ${ }^{35} \mathrm{y}$ que aparece posteriormente con el título de Il finto marchese y, finalmente, con ambos rótulos: Il finto marchese overo Le gelose cautele. Comedia di M. M. B. Accad. Affinato, Roma, Michel'Ercole, 1676 ${ }^{36}$.

33 Calcolone, Ettore, Gli effetti overo Gli eccessi della cortesia, In Napoli et in Bologna, Per Gioseffo Longhi, 1670, f. A3r. Ejemplar consultado: Milán, Biblioteca Nazionale Braidense, Racc. Dram. 0574 .

${ }^{34}$ Cf. DBI, VI, pp. 790-791.

35 Cit. Sanesi, La commedia, II, p. 199.

36 Franchi informa: «Prima edizione: Le gelose cautele (In Firenze, nella Stamp. di S. A. S., 1668). La commedia è imitazione di un'opera di Francisco de Rojas Zorrilla. L'autore è Mattia Maria Bartolomei. Con il titolo originale la commedia ebbe altre tre edizioni: In Bologna, per il 
La fuente es una obra maestra del ingenio toledano, cuya doble titulación tendrá variada fortuna ${ }^{37}$ : Donde no hay agravios no hay celos y amo y criado. En el ejemplar consultado de $1676^{38}$, en el f. A ${ }^{3}$ r se señala que la escena se representa en Milán y que los personajes son «D. Fernando Padre d' / Isabella/ Lisetta loro serua/ D. Gio. D'Aluarado/ Leonora sua sorella/ Brandello loro seruitore/ Enrico nipote di D. Fernando/ Florante suo seruitore».

\section{SIGLO XVIII}

La reacción ilustrada a las modalidades teatrales anteriores (la comedia española y la Commedia dell'arte) diluye los rastros de la influencia dramática española y, en cambio, aumenta la presencia francesa. Como parámetro indicativo de esta tendencia, piénsese en la colección teatral enhebrada por Ottaviano Diodati en 12 tomos con el fin de divulgar una preceptiva dramática actual: Biblioteca teatrale italiana scelta e disposta da Ottaviano Diodati, patrizio lucchese. Con un suo capitolo in verso per ogni tomo, correlativo alle cose teatrali, per servire di Trattato completo di Drammaturgia, Lucca, Giovanni della Valle, 1762, 12 vols. Los volúmenes, que, por lo general, contienen una sección trágica y otra cómica, proponen tragedias, comedias, obras para música, intermedios cómicos de varios autores: el mismo editor, Scipione Maffei, Michelangelo Buonarroti, Ludovico Savioli, Pietro Metastasio, Girolamo Gigli, Vittorio Amedeo Cigna, Pietro Chiari, Carlo Innocenzo Frugoni, Marco Coltellini y Domenico Serafini, Carlo Goldoni, etc. Los textos anunciados como adaptaciones o traducciones de obras extranjeras enlazan con el teatro francés original (Racine, De la Chaussé, Crebillon, De la Motte, etc.) o con la cultura francesa como intermediaria de otras literaturas (es el caso de Il caffe o La scozzese, comedia moderna inglesa que fue traducida al francés y de ahí al italiano). Por consiguiente, el teatro español decae a favor de otro sistema dramático y otro gusto, pero con el siglo siguiente conseguirá ser reivindicado.

\footnotetext{
Manolessi, 1669; In Bologna, 1676. Presso Domenico Maria Feroni, Ad istanza di Gioseffo Longhi (tratta dall'edizione romana); In Bologna, nella Stamperia del Longhi, 1694» (Franchi, Drammaturgia romana..., p. 492). Cf. Allacci, Leone, Drammaturgia di Leone Allacci accresciuta $e$ continuata fino all'anno 1755, Venezia, Giabattista Pasquali, 1755 (reimpr.: Torino, Bottega d'Erasmo, 1966), p. 388.

37 Rojas Zorrilla, Francisco de, Donde hay agravios no hay celos. Abrir el ojo, ed. Felipe B. Pedraza Jiménez y Milagros, Rodríguez Cáceres, Madrid, Castalia, 2005 , pp. 25-27.

38 Conservado en Roma, Biblioteca Nazionale, 35.8.A.11.2.
} 
La recepción del teatro de Francisco de Rojas Zorrilla en Italia. Algunas anotaciones.

\section{SIGLO XIX}

Es sabido que al Romanticismo se debe la moderna recuperación de la dramaturgia española aurisecular. También en Italia se aprecia este retorno. En los años cuarenta Pietro Monti, conocido por sus traducciones calderonianas, vuelca al italiano el drama Del rey abajo ninguno de Rojas Zorrilla en Il Garzia del Castagneto que se publica en una edición en dos tomos de comedias selectas, mayoritariamente calderonianas ${ }^{39}$. Algunos años después se confecciona una edición colectiva del teatro español. Una postura polémica anima a su traductor: dar a conocer «i capolavori del primo, del più ricco teatro d'Europa», para contrarrestar la

strabocchevole quantità di assurdi drammi, usciti dalla scuola moderna francese, ove nè profondità di concetti, né sfoggio d'immaginazione, né spontaneità di dialogo, $t^{\prime}$ è dato di rinvenir mai... ${ }^{40}$

La defensa del teatro español se conduce, por un lado, señalando que fueron los pedantes del siglo XVIII quienes lo condenaron a causa de sus malos imitadores; y, por el otro, proporcionando al lector una espléndida y extensa selección de obras dramáticas de los siglos XV-XVIII ${ }^{41}$. Del teatro de Rojas

39 Rojas Zorrilla, Francesco di, Il Garzia del Castagneto, en Commedie ..., trad. Pietro Monti, Milano, Della Società Tipog. de' Classici Italiani, 1938-1940, 2 vols., II, pp. 317-379. De los títulos anunciados en las portadas (I: Amore dopo la morte. La devozione della croce. L'aurora di Copacabana; II: Il pozzo di San Patrizio. Il principe costante. La vita è un sogno. Il Tetrarca de Calderón e Il Garzia del Castagneto de Rojas) se especifican en las portadillas internas los últimos dos (Il maggior mostro la gelosia y Salvo il re, nessuno e contadino onorato, Garzia del Castagneto).

40 Teatro scelto spagnuolo antico e moderno. Raccolta dei migliori drammi, commedie e tragedie, trad. Giovanni La Cecilia, Torino, Unione Tipografico-Editrice, 1857-1859, 8 vols., VI, p. 271.

41 En esta colección se publica una media de seis piezas teatrales por volumen. Según las portadillas italianas, el reparto es el siguiente: I. Gl'inganni, commedia in 5 atti e in prosa di Lope de Rueda, 1556; Cornuto e contento, proverbio di Lope de Rueda, 1556; Celestina, novella drammatica, o La tragicommedia di Calisto e Melibea, scritta da Rodrigo Cota e Ferdinando Royas [sic.], 1571; Il sacco di Roma o La morte di Borbone, di Giovanni della Cueva, 1579; Ines pietosa, tragedia di Geronimo Bermudez conosciuto col nome di Antonio de Silva, 1577; Ines coronata, tragedia di Geronimo Bermudez conosciuto col nome di Antonio de Silva, 1577; I crociati all'assedio di Lavaur, tragedia storica di Alonso Cisneros, 1573; Il mercadante innamorato, commedia di Aguilar, 1600; II. I male maritati di Valenza, commedia di Guillen de Castro, 1600; La burlata, commedia di Michel de Cervantes Saavedra stampata nell'anno 1610; Il geloso, commedia di don Alfonso Uz de Velasco, 1600; La Numanzia, tragedia di Michele Cervantes, 1586; La schiava del suo galante, commedia di Lope de Vega, 1605; III. La bella brutta, commedia di Lope de Vega, 1612; Il cane dell'ortolano, commedia di Lope de Vega, 1620; Il nuovo mondo scoperto da Cristoforo Colombo, dramma di Lope de Vega, 1627; La vita è un sogno, commedia di Lope de Vega [sic.], 1628; A segreta offesa celata vendetta, tragicommedia di Calderon de la Barca, 1622; La divozione della croce, commedia di Calderon de la Barca, 1641; L'alcalde di Zalamea, commedia di Calderon de la Barca, 1650; IV. Le armi della bellezza, commedia di Calderon de la Barca, 1650; Il pudico Giuseppe delle 
Zorrilla se eligen tres obras relevantes: Del rey abajo ninguno (Don Garzia dal Castagneto ovvero Dal Re in fuori nessuno. Dramma di don Francesco de Royas [sic.] ${ }^{42}$ ), Donde hay agravios no hay celos (Padrone e servo ovvero Dov'è l'onta non è la gelosia. Dramma di don Francesco de Royas [sic.] $]^{43}$ ) y Entre bobos anda el juego (Da galeotto a marinaro. Commedia di D. Francesco de Royas [sic.] $\left.{ }^{44}\right)$.

Esta selección se justifica, fundándose en la variedad de géneros y la calidad dramática, con palabras rotundas:

donne, commedia di Calderon de la Barca, 1630; Don Gil dai calzoni verdi [pero después del dramatis personae: Don Gil dalle calze verdi], commedia di Gabriele Tellez (vulgo Tirso de Molina), 1600; Il convitato di pietra, dramma di Gabriele Tellez (vulgo Tirso de Molina), 1621; La prudenza delle donne, dramma di Gabriel Tellez (vulgo Tirso de Molina), 1610; Sdegno contro sdegno, commedia di don Agostino Moreto, 1650; V. Il valente giudice [pero en los titulillos a veces Il giudice valente], dramma di don Agostino Moreto, 1677; Don Diego il damerino, commedia di don Agostino Moreto, 1770 [sic.]; I fiori di don Giovanni o Ricco e povero cambiati, commedia di Lope de Vega, 1620; Se le donne non vedessero, commedia di Lope de Vega, 1620; L'onorato fratello, tragedia di Lope de Vega, 1615; I pazzzi [sic.] di Valenza, commedia di Lope de Vega, 1625; VI. Il tessitore di Segovia, azione drammatica di don Giovanni Ruiz d'Alarcon, 1730 [sic], parte prima; Il tessitore di Segovia, azione drammatica di don Giovanni Ruiz d'Alarcon, 1730 [sic], parte seconda; Acquistare amici, commedia di don Giovanni Ruiz d'Alarcon; Le pareti odono, commedia di don Giovanni Ruiz d'Alarcon; La verità sospetta, commedia di don Giovanni Ruiz d'Alarcon; D. Garzia dal Castagneto ovvero Del re in fuori nessuno, dramma di don Francesco de Royas; Padrone e servo ossia Dov'è l'onta non è la gelosia, dramma di don Francesco de Royas; VII. Da galeotto a marinaro, commedia di don Francesco de Royas; Prima l'onore poi la vita, commedia di don Giovanni Perez di Montalavan, 1628; Marta la bacchettona ovvero La pia innamorata, commedia d'incerto autore, 1631; Mi chiamo Lorenzo ossia Il carbonaro di Toledo, commedia di Matteo Fragoso, 1640; Dal disprezzo il contento, commedia di Matteo Fragoso, 1670; Regnar dopo la morte, dramma di Luigi Velez di Gnevara [sic], 1630; VIII, Il diavolo predicatore ossia Il più contrario amico, commedia di don Luigi di Belmonte; Il Cid ossia Il figlio che onora il padre, commedia di don Giovanni Battista Bramante [sic]; Il Conte $d^{\prime}$ Essex, tragedia di Filippo IV re di Spagna; L'amore alla moda, commedia di don Antonio de Solis; Il sì delle fanciulle, commedia di Leandro Fernandez de Moratin; Pelagio, tragedia di don Emanuele Giosuè Quintana.

${ }^{42}$ Teatro scelto spagnuolo..., VI, pp. 269-315.

43 Teatro scelto spagnuolo..., VI, pp. 317-375. El traductor informa en nota: «Questo dramma fu uno dei più fortunati in sulla scena spagnuola. Gli applausi che ottenne ogni volta che fu rappresentato stimolarono un letterato francese a tradurlo in quell'idioma, sotto il titolo appunto di Padrone e servo, e in Francia fu accolto con pari entusiasmo» (Teatro scelto spagnuolo..., VI, p. 317).

44 Teatro scelto spagnuolo..., VII, pp. 5-60. El traductor defiende la elección del título italiano en estos términos: «Ecco il titolo originale: Entre bobos anda el juego, ciò che significa: Fra gonzi va il giuoco. Queste parole sono però poste in bocca ad un uomo sciocco e presuntuoso, siccome il vedranno i nostri lettori. Or un baggeo che vuole spacciarsi per un uomo accorto non confessa giammai d'essere gonzo, e se ciò dice, il dice a mo' d'ironia. Per le quali cose abbiamo sostituito al titolo spagnuolo un motto proverbiale italiano, che il traduce a capello» (Teatro scelto spagnuolo..., VI, p. 5). 
La recepción del teatro de Francisco de Rojas Zorrilla en Italia. Algunas anotaciones.

Don Garzia del Castagneto è un dramma storico dei più stupendi che possa vantar l'arte in tutta Europa. Padrone e servo, stassi fra il dramma e la commedia. Da galeotto a marinaro, è un lavoro tutto comico. $\mathrm{E}^{\prime}$ sono tre mirabili lavori. Roxas è in tutte le sue produzioni originale, tanto nello stile, quanto in isvolgere la tela. ${ }^{45}$

Solamente tres piezas de Rojas Zorrilla, pero heterogéneas y bien labradas dramáticamente. Una presencia que puede parecer insignificante, sobre todo si se compara con los grandes Lope (7 obras) y Calderón (6 obras); pero que debe apreciarse, porque se equipara o supera a la de otros dramaturgos (Tirso, 3 obras; Moreto, 3; Matos Fragoso, 2; Solís, 2) y, sobre todo, coloca en una buena posición dentro del canon teatral aurisecular a un autor que, todavía, no se ha reconocido como es debido.

45 Teatro scelto spagnuolo..., VII, p. 5. 


\section{APÉNDICE}

LISTADO DE LAS TRADUCCIONES, REFUNDICIONES, SCENARI, ETC. DEL TEATRO DE ROJAS ZORRILLA (se señalan con un signo de interrogación entre paréntesis las hipótesis avanzadas a la espera de confirmación)

Baltasara, La

Rospigliosi, Giulio, La Baldasara o La comica del cielo. Varios mss.

Caín de Cataluña, El

Anónimo, La giustizia catalana. Roma, Biblioteca Casanatense, ms. 4186.

Anónimo, Il fratricida crudele, le finte caccie con Bertolino impiccato. Roma, Biblioteca Casanatense, ms. 4186.

Catalán Serrallonga, El

Napolioni, Marco, Il gran catalan Sacralonga. Sin localizar.

Casarse por vengarse

Cicognini, Giacinto Andrea, Il maritarsi per vendetta. Varias ediciones y ejemplares.

Cicognini, Giacinto Andrea, La forza del fato o Il matrimonio nella morte. Varias ediciones y ejemplares.

Napolioni, Marco, Il maritarsi per vendetta. (?) Sin localizar.

Del rey abajo ninguno y labrador más honrado García del Castañar

Monti, Pietro, Salvo il re, nessuno e contadino onorato, Garzia del Castagneto, 18381840.

La Cecilia, Giovanni, Don Garzia dal Castagneto overo Dal re in fuori nessuno, 185758.

Donde hay agravios no hay celos y amo criado

Napolioni, Marco, Gli aggravi trionfanti della gelosia. (?) Sin localizar.

D'Orso, Angela, A chi l'Onor li offende è gran pazzia se sente le puntur di gelosia. (?) Sin localizar.

Bartolommei, Mattias Maria, Le gelose cautele o Il finto marchese. Varias ediciones y ejemplares.

La Cecilia, Giovanni, Padrone e servo, 1857-1858.

Entre bobos anda el juego

La Cecilia, Giovanni, Da galeotto a marinaro, 1857-1858. 
La recepción del teatro de Francisco de Rojas Zorrilla en Italia. Algunas anotaciones.

falso profeta Mahoma, El

Napolioni, Marco, Il Macometto. Sin localizar.

más impropio verdugo por la más justa venganza, El

D’Orso, Angela, Il più improprio carnefice per la più giusta vendetta. Sin localizar.

No hay ser padre siendo rey

Celano, Carlo, Non è padre essendo re. Varias ediciones y ejemplares.

Obligados y ofendidos

Celano, Carlo, Gli effetti overo gli eccessi della cortesia. Varias ediciones y ejemplares.

Peligrar en los remedios

Napolioni, Marco, Il pericolo ne' rimedi. Sin localizar.

Persiles y Segismunda

Napolioni, Marco, Persile e Sigismonda. Sin localizar.

Santa Isabel, reina de Portugal

Cicognini, Giacinto Andrea, L'innocenza calunniata, overo la regina di Portogallo Elisabetta la santa (Bologna, Gioseffo Longhi, s. a. [probl. Entre 1655 y 1691]) 